\title{
Peculiar Energy-Scattering Angle Dependence of the Alignment Angle of Atoms Excited by Electron Impact
}

\author{
M. Piwiński, Ł. KŁosowski, S. Chwirot* \\ Institute of Physics, Faculty of Physics, Astronomy and Informatics, Nicolaus Copernicus University in Toruń, \\ Grudziądzka 5, 87-100 Toruń, Poland \\ (Received February 21, 2018; in final form June 5, 2018)

\begin{abstract}
Some peculiar structures in scattering angle-collision energy dependence of the alignment angle parameter have been found in data sets for zinc and cadmium atoms. They are accompanied by zeros of the shape parameter value, where the maximum angular momentum transfer occurs. The reported structures are also predicted in data for other atoms with two valence electrons. There is a need for theoretical predictions on impact energy and scattering angles corresponding to such conditions.
\end{abstract}

DOI: 10.12693/APhysPolA.134.RK.134.2.1-1

PACS/topics: 34.80.-i, 34.80.Dp

\section{Introduction}

A complete description of a scattering process of two quantum objects requires determination of complex scattering amplitudes depending on both the scattering angle and the collision energy. Electron impact excitation from $S$ to $P$ atomic state can be fully characterised by values of a differential cross-section and of a set of parameters such as the widely used electron impact coherence parameters (EICP): alignment angle $\gamma$, shape parameter $P_{L}$ and angular momentum transfer $L_{\perp}$ [1]. The so-called complete experiments on inelastic electron-atom collisions allow determination of the EICPs i.e. not only absolute values of scattering amplitudes that can be obtained from the relevant cross-sections, but also of their mutual phases. The latter determine the alignment angle of the excited atoms, a quantity directly measured by analysis of either angular distribution or polarisation of the fluorescence.

Measurements of the EICPs are generally timeconsuming especially at larger scattering angles where weeks of data integration is a common feature. Therefore, the experimental data are sparse, usually available for a limited set of few collisional energies. The same applies to numerical studies, usually covering the impact energies relevant for experimental research.

In spite of limited amount of data, attempts have been made to formulate broader interpretation of the EICPs in terms of "propensity rules" [2-4]. Those studies focused mainly on analysis of $L_{\perp}$ parameter, while less attention was paid to the alignment angle function due to its regular and quite predictable character. However, our analysis of available experimental and theoretical results demonstrates existence of special scattering conditions (scattering energy and angle), where unusual behaviour

\footnotetext{
* corresponding author; e-mail: Mariusz.Piwinski@fizyka.umk.pl
}

of this function may be expected with relatively narrow features. In some cases the discrepancies between EICP data sets could be explained by a slight shift in collision energy or scattering angle domain $[5,6]$.

\section{Electron impact coherence parameters}

The complete definition of EICPs can be found in the seminal review article of Andersen et al. [1]. Briefly, if the atom is collisionally excited from $S_{0}$ ground to $P_{1}$ excited state, it can be described as a coherent superposition of the magnetic sub-states. In the so-called natural reference frame with the quantization axis perpendicular to the scattering plane only two of the sub-states yield non-zero contributions to the superposition and the excited $P$ state of the atom can be described as

$$
|P\rangle=a_{-1}\left|m_{j}=-1\right\rangle+a_{+1}\left|m_{j}=+1\right\rangle,
$$

where the complex coefficients $a_{ \pm 1}$ represent the relevant excitation amplitudes.

The angular part of the electron charge cloud of the atom can be written in standard spherical coordinates as

$$
|\Psi(\vartheta, \varphi)|^{2}=\frac{3}{8 \pi} \sin ^{2} \vartheta\left(1+P_{L} \cos (2 \varphi-2 \gamma)\right),
$$

where $P_{L}$ is shape parameter and $\gamma$ is alignment angle $[1,4]$. The two parameters are related to the excitation amplitudes by following formulae:

$$
\begin{aligned}
& \gamma=\frac{1}{2} \arg \left(-a_{-1}^{*} a_{+1}\right), \\
& P_{L}=2\left|a_{-1}^{*} a_{+1}\right| .
\end{aligned}
$$

Another parameter needed for a complete description of the collision process is related to angular momentum transfer and defined as:

$$
L_{\perp}=\left|a_{+1}\right|^{2}-\left|a_{-1}\right|^{2} .
$$

In the case of a fully coherent scattering process the parameters $L_{\perp}$ and $P_{L}$ fulfil equation

$$
L_{\perp}^{2}+P_{L}^{2}=1 .
$$


The main reason of common acceptance of the EICPs has been their direct relation to atomic observables and to geometrical properties of the electron cloud angular distribution of the excited atoms (see Fig. 1).

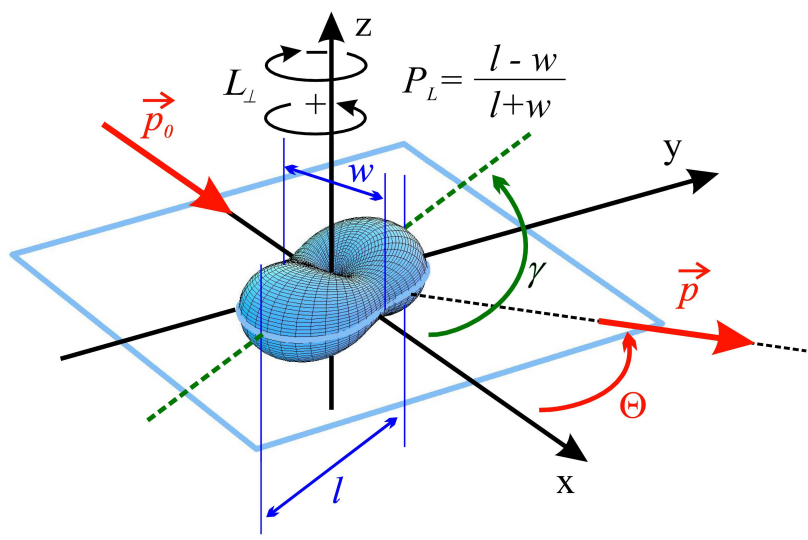

Fig. 1. Example of angular distribution of the electron charge cloud of an atomic $P$ state. The $\vec{p}_{0}$ and $\vec{p}$ symbols represent momenta of incoming and scattered electron, $\Theta$ is the scattering angle, $\gamma$ represents the alignment angle, and $L_{\perp}$ is the angular momentum transfer parameter. The shape parameter $P_{L}$ can be related to the length $l$ and width $w$ of the cloud.

According to Eqs. (3) and (4), $P_{L}$ and $\gamma$ parameters can be seen as representing polar components of a complex product of the scattering amplitudes $a_{-1}^{*} a_{+1}$ (the $\frac{1}{2}$ and 2 factors are conventional). The product is a function of the scattering angle and impact energy

$$
a_{-1}^{*} a_{+1}=f(\Theta, E) \text {. }
$$

If any of the two scattering channels is completely closed, the electron charge cloud is rotationally symmetric with respect to the $z$ axis $\left(P_{L}=0\right)$ and the alignment angle parameter $\gamma$ becomes indefinable, while the angular momentum transfer parameter reaches the maximum value $( \pm 1)$. Moreover, in a vicinity of $P_{L}=0$ rapid changes of $f(\Theta, E)$ for the $E$ and $\gamma$ values can be expected resulting from changes in phase difference between $a_{-1}$ and $a_{+1}$. Therefore, such regions seem of special interest for both experimental studies and theoretical modelling of the inelastic electron-atom collisions.

In the case of the excitation process of interest the alignment angle parameter is a continuous function of two scattering amplitudes (see Eq. (3)), thus adding or subtracting $180^{\circ}$ is meaningless from a point of view of physical interpretation. Its experimental and theoretical values are typically presented in the range of $\left(-90^{\circ}, 90^{\circ}\right)$, and from now on, we shall restrict the discussion to this interval only. Figure 2 shows an example of the predicted behaviour of the alignment angle as a function of both the scattering angle and the electron impact energy in the neighbourhood of arbitrarily chosen values of these parameters.

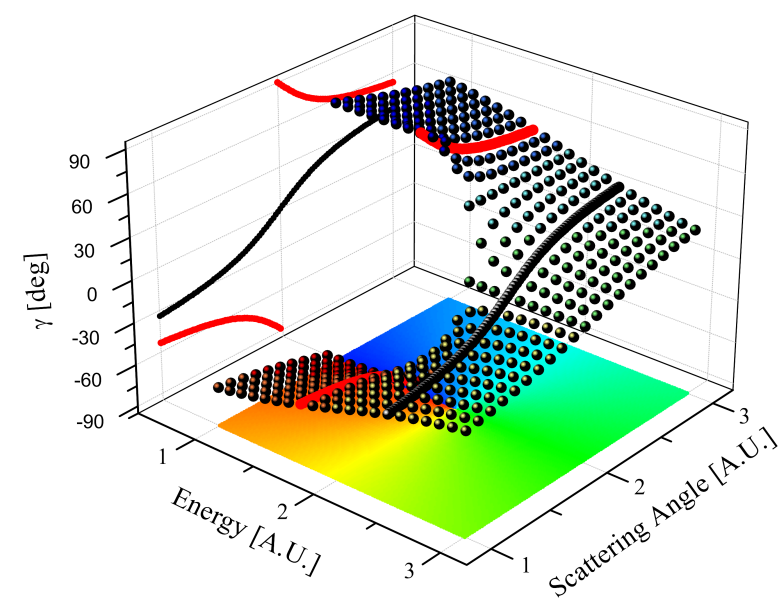

Fig. 2. Illustration of the predicted behaviour of the alignment angle for the impact energy and the scattering angle in the neighbourhood of arbitrarily chosen values of these parameters (see Eqs. (3) and (7)). The 2D colour map represents the same function (from $-90^{\circ}$ red to $+90^{\circ}$ blue). Additionally $2 \mathrm{D}$ projections are presented for two values of the impact energy corresponding to two different modes in changes of the alignment angle with the scattering angle: discontinuous descending (red points) and continuous ascending (black points).

It can be seen that there are characteristic patterns of changes of the alignment angle with the scattering angle at a fixed impact energy that can be summarized as follows: (i) continuous ascending (black points in Fig. 2), (ii) continuous descending, (iii) discontinuous descending (red points in Fig. 2) and (iv) discontinuous ascending. As illustrated in the 3D plots (Fig. 2) values of the alignment angle are distributed on a helical surface.

\section{Analysis of alignment angle data}

Typical EICPs dependence on the electron scattering angle does not change significantly with slight changes of the impact energy. However, as found at a detailed analysis of the existing data sets, there are exceptions from this rule. On several occasions it was found that the alignment angle functions change rapidly their character for two relatively close energies (see the $2 \mathrm{D}$ projections presented in Fig. 2).

One of the examples is data set for e- $\mathrm{Zn}$ scattering at 60, 80, and $100 \mathrm{eV}$ presented in Fig. 3 [7-10]. There is a clear difference between the patterns of the alignment angle changes at scattering angles around $150^{\circ}$ at the impact energies of $80 \mathrm{eV}$ and $100 \mathrm{eV}$ predicted by both the convergent close coupling model (CCC) and the relativistic distorted wave approximation (RDWA). The alignment angle function is clearly continuous at $100 \mathrm{eV}$ (continuous descending) while for $80 \mathrm{eV}$ it reaches values of $-90^{\circ}$ and $90^{\circ}$ (discontinuous ascending). Thus, considering the impact energies between $80 \mathrm{eV}$ and $100 \mathrm{eV}$ one can expect a helical structure to occur for the scattering angle close to $150^{\circ}$ (at impact energy values corresponding to $P_{L}=0$ ). Moreover, for collision energy $60 \mathrm{eV}$ the 
alignment angle function has again continuous descending character (as in case for $100 \mathrm{eV}$ ), therefore second helical structure between those energies is expected.

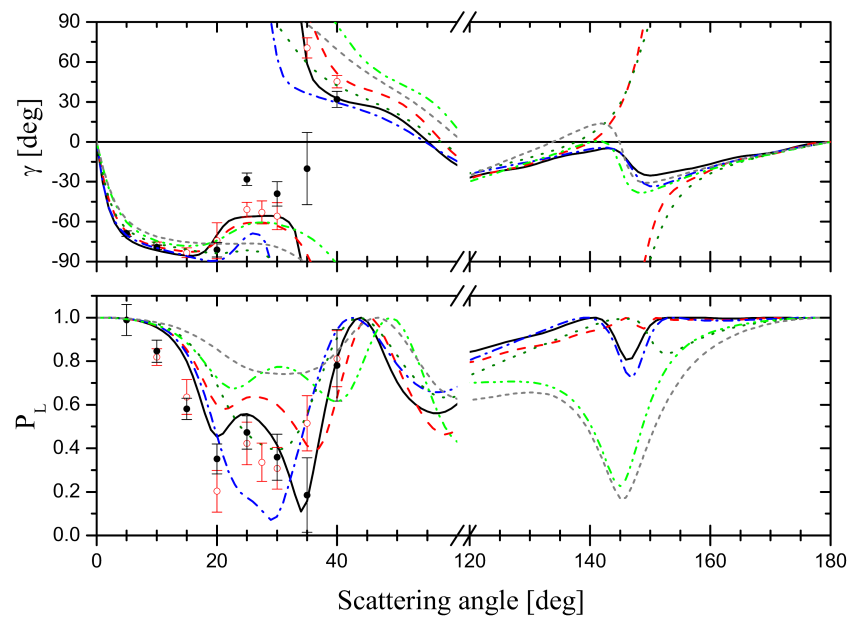

Fig. 3. Alignment angle and shape parameter functions for electron impact $4^{1} P_{1}$ excitation of $\mathrm{Zn}$. The theoretical CCC data $(100 \mathrm{eV}$ denoted as $(-), 80 \mathrm{eV}(--)$, $60 \mathrm{eV}(-\cdots))$ and RDWA results $(100 \mathrm{eV}(-\cdot-), 80 \mathrm{eV}$ $(\cdots), 60 \mathrm{eV}(--))$ are presented together with experimental data $(100 \mathrm{eV}(\bullet), 80 \mathrm{eV}(\circ))[7-10]$.

Similar pattern (discontinuous and continuous functions) can be observed for the experimental data at the scattering angle of $c a .30^{\circ}$, however, it is not reproduced by numerical data. To explain discrepancy between experiment and theories, one should take into account the $P_{L}$ function presented in Fig. 3 . The $P_{L}$ value at the scattering angle of $c a .30^{\circ}$ is very close to zero at the impact energy of $100 \mathrm{eV}$. Thus one can expect the rapid change of the gamma function behaviour in theoretical predictions for a little bit larger collision energy than $100 \mathrm{eV}$, for which it was observed in the experiment. In this area the helical course of changes of the alignment angle is expected. Therefore according to Fig. 2 in a proximity of the central point of this structure (in this case neighbourhood of $100 \mathrm{eV}$ ) small changes in the impact energy result in significant differences in $\gamma$.

Moreover, the relatively shallow minima of $P_{L}$ predicted by both the CCC and RWDA theories (for $100 \mathrm{eV}$ and $80 \mathrm{eV}$ impact energies) around the scattering angle of $150^{\circ}$ indicate a region of helical structure located between, but at a reasonable distance from either of the two energy values investigated so far.

Similar pattern is present in the theoretical data reported for the excitation of $\mathrm{Cd}$ atoms (Fig. 4) [6, 11, 12]. Around the scattering angle of $c a .140^{\circ}$ the shape of the alignment angle function significantly changes for different impact energies. Moreover, the theoretical predictions based on the CCC and RDWA approaches are quite inconsistent for the impact energies of interest.

The CCC model indicates a presence of one helical structure located at the impact energy value between
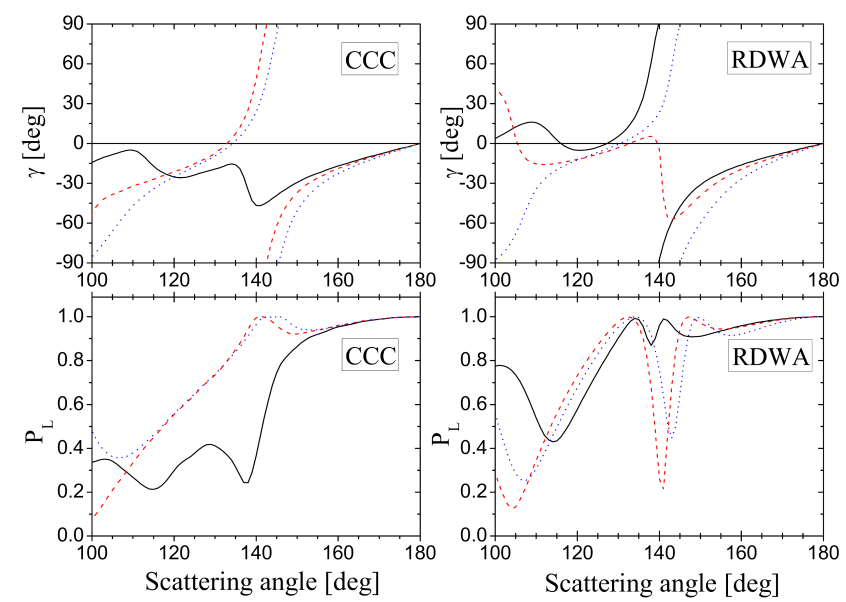

Fig. 4. Alignment angle and shape parameter functions for the electron impact $5^{1} P_{1}$ excitation of $\mathrm{Cd}$ for incident energies: $100 \mathrm{eV}(-), 80 \mathrm{eV}\left(-_{-}^{-}\right)$ and $60 \mathrm{eV}(\cdots)[7-9]$.

$80 \mathrm{eV}$ and $100 \mathrm{eV}$, while the RDWA calculations suggest existence of two such structures: one between $100 \mathrm{eV}$ and $80 \mathrm{eV}$ and the second between $60 \mathrm{eV}$ and $80 \mathrm{eV}$. As could be expected, all these structures are associated with deep minima in $P_{L}$ (one according to the CCC and two for the RDWA predictions) shown in Fig. 4.

In spite of relatively sparse EICPs data, similar patterns of the changes of the alignment angles (discontinuous and continuous) for two neighbouring collision energies, indicating a presence of the helical structures can also be found for other atomic species (see Table I). The reported structures are predicted for different impact energies and a broad range of the scattering angles (see for example data for magnesium illustrated in Fig. 5).

TABLE I

Regions (impact energies and electron scattering angles) of peculiar changes of the alignment angle observed for electron impact excitation of different atomic species. The scattering angle values listed are indicatory.

\begin{tabular}{c|c|c|l}
\hline \hline Atom & Energy range $[\mathrm{eV}]$ & Scatt. angle $\Theta\left[^{\circ}\right]$ & Ref. \\
\hline $\mathrm{Mg}$ & $10-20$ & 70 & {$[13]$} \\
$\mathrm{Mg}$ & $20-40$ & 50 & {$[13,14]$} \\
$\mathrm{Mg}$ & $50-100$ & 100 & {$[15]$} \\
$\mathrm{Ca}$ & $10-12$ & 100 & {$[5]$} \\
$\mathrm{Ca}$ & $35-45$ & 140 & {$[16,17]$} \\
$\mathrm{Ca}$ & $45-55$ & 20 & {$[17-19]$} \\
$\mathrm{Zn}$ & $80-100$ & 30 & {$[7-9]$} \\
$\mathrm{Zn}$ & $80-100$ & 150 & {$[7-9]$} \\
$\mathrm{Sr}$ & $30-58$ & 55 & {$[20]$} \\
$\mathrm{Cd}$ & $60-80$ & 140 & {$[12]$} \\
$\mathrm{Cd}$ & $80-100$ & 140 & {$[6,11,12]$} \\
$\mathrm{Ba}$ & $15-20$ & 80 & {$[21]$} \\
$\mathrm{Ba}$ & $20-47$ & 20 & {$[22]$} \\
$\mathrm{Ba}$ & $36-50$ & 140 & {$[21]$} \\
$\mathrm{Ba}$ & $50-80$ & 40 & {$[21]$}
\end{tabular}



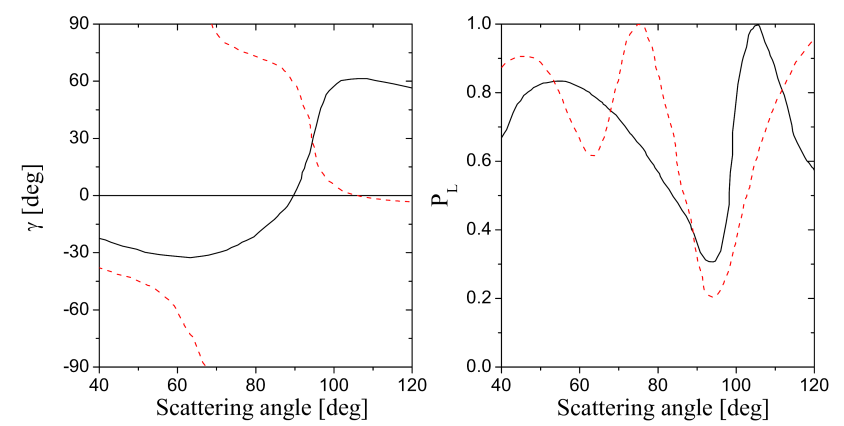

Fig. 5. Alignment angle and shape parameter functions for the electron impact $(3 s 3 p)^{1} P^{\circ}$ excitation of $\mathrm{Mg}$ for incident energies: $10 \mathrm{eV}(-)$ and $20 \mathrm{eV}(--)$ [13].

\section{Conclusions}

Theoretically predicted helical structures of the $\gamma$ functions were found analysing existing EICPs data for zinc and cadmium. Similar patterns were also observed in the scattering angle-impact energy dependence of the alignment angle for other atoms with two valence electrons. Available sparse data sets do not allow for the exact determination of locations of those helical structures. It is, however, possible to define intervals of the collision energies and of the scattering angles where they might be found. Studies of the collisional dynamics within such regions could give a better insight into the phase relation between scattering amplitudes. Due to the great progress of computational techniques in recent years, it should be possible to find the helical structures on threedimensional maps of the EICPs prepared with sufficiently high resolution. Such data could be energetically and angularly convoluted to emulate finite experimental resolutions in both energy and scattering angle domains.

\section{References}

[1] N. Andersen, J. W. Gallagher, I. V. Hertel, Phys. Rep. 165, 1 (1988).

[2] M. Kohmotot, U. Fano, J. Phys. B 14, L447 (1981).
[3] D.H. Madison, K.H. Winters, Phys. Rev. Lett. 47, 1885 (1981).

[4] N. Andersen, K. Bartschat, Polarization, Alignment, and Orientation in Atomic Collisions, Vol. 96 of Atomic, Molecular, Optical and Plasma Physics, Springer Int. Publ., Cham 2017.

[5] A. Knight-Percival, S. Jhumka, M. Hussey, A.J. Murray, J. Phys. B 44, 105203 (2011).

[6] M.J. Berrington, C.J. Bostock, D.V. Fursa, I. Bray, R.P. McEachran, A.D. Stauffer, Phys. Rev. A 85, 042708 (2012).

[7] M. Piwiński, Ł. Kłosowski, D. Dziczek, S. Chwirot, T. Das, R. Srivastava, A.D. Stauffer, C.J. Bostock, D. Fursa, I. Bray, Phys. Rev. A 86, 052706 (2012).

[8] T. Das, L. Sharma, R. Srivastava, A.D. Stauffer, Phys. Lett. A 378, 641 (2014).

[9] M. Piwiński, Ł. Kłosowski, D. Dziczek, S. Chwirot, D.V. Fursa, I. Bray, Phys. Rev. A 91, 062704 (2015).

[10] M. Piwiński, Ł. Kłosowski, S. Chwirot, D. V. Fursa, I. Bray, T. Das, R. Srivastava, J. Phys. B 51, 085002 (2018).

[11] M. Piwiński, D. Dziczek, R. Srivastava, M. Grądziel, S. Chwirot, J. Phys. B 35, 3821 (2002).

[12] M. Piwiński, D. Dziczek, Ł. Kłosowski, R. Srivastava, S. Chwirot, J. Phys. B 39, 1945 (2006).

[13] D.V. Fursa, I. Bray, Phys. Rev. A 63, 032708 (2001).

[14] D.O. Brown, A. Crowe, D.V. Fursa, I. Bray, K. Bartschat, J. Phys. B 38, 4123 (2005).

[15] G.D. Meneses, C.B. Pagan, L.E. Machado, Phys. Rev. A 41, 4740 (1990).

[16] A. Murray, D. Cvejanovic, J. Phys. B 36, 4889 (2003).

[17] M. Hussey, A. Murray, W. MacGillivray, G.C. King, J. Phys. B 41, 055202 (2008).

[18] D. Dyl, D. Dziczek, M. Piwiński, M. Grądziel, R. Srivastava, R. Dygdała, S. Chwirot, J. Phys. B 32, 837 (1999).

[19] D.V. Fursa, I. Bray, J. Phys. B 41, 145206 (2008).

[20] H.J. Beyer, H. Hamdy, E.I.M. Zohny, K.R. Mahmoud, M.A.K. El-Fayoumi, H. Kleinpoppen, J. Abdallah Jr., R.E.H. Clark, G. Csanak, Z. Phys. D 30, 91 (1994).

[21] D.V. Fursa, I. Bray, Phys. Rev. A 59, 282 (1999).

[22] P.W. Zetner, Y. Li, S. Trajmar, Phys. Rev. A 48, 495 (1993). 